\title{
Cost-Effectiveness Analysis of High-Efficiency Hemodiafiltration Versus Low-Flux Hemodialysis Based on the Canadian Arm of the CONTRAST Study
}

\author{
Renee Lévesque $^{1,2}$ - Daniele Marcelli ${ }^{3}$ Héloïse Cardinal ${ }^{1,4} \cdot$ Marie-Line Caron $^{2} \cdot$ \\ Muriel P. C. Grooteman ${ }^{5,6} \cdot$ Michiel L. Bots ${ }^{7} \cdot$ Peter J. Blankestijn ${ }^{8}$. \\ Menso J. Nubé (5,6 $^{-}$Aileen Grassmann ${ }^{3} \cdot$ Bernard Canaud $^{3} \cdot$ Afschin Gandjour $^{9}$
}

Published online: 14 June 2015

(C) The Author(s) 2015. This article is published with open access at Springerlink.com

\begin{abstract}
Aim The aim of this study was to assess the cost effectiveness of high-efficiency on-line hemodiafiltration (OLHDF) compared with low-flux hemodialysis (LF-HD) for patients with end-stage renal disease (ESRD) based on the Canadian (Centre Hospitalier de l'Université de Montréal) arm of a parallel-group randomized controlled trial (RCT), the CONvective TRAnsport STudy.

Methods An economic evaluation was conducted for the period of the RCT (74 months). In addition, a Markov state transition model was constructed to simulate costs and health benefits over lifetime. The primary outcome was costs per quality-adjusted life-year (QALY) gained. The
\end{abstract}

Daniele Marcelli

daniele.marcelli@fmc-ag.de

Afschin Gandjour

A.Gandjour@fs.de

1 Department of Nephrology, Centre Hospitalier de l'Université de Montréal, Montréal, Canada

2 St. Luc Hospital, Montréal, Canada

3 EMEALA Medical Board, Fresenius Medical Care, Bad Homburg, Germany

4 Notre-Dame Hospital, Montréal, Canada

5 Department of Nephrology, VU University Medical Center, Amsterdam, The Netherlands

6 Institute for Cardiovascular Research, VU University Medical Center, Amsterdam, The Netherlands

7 Julius Center for Health Sciences and Primary Care, University Medical Center Utrecht, Utrecht, The Netherlands

8 Department of Nephrology, University Medical Center, Utrecht, The Netherlands

9 Frankfurt School of Finance and Management, Frankfurt Am Main, Germany analysis had the perspective of the Quebec public healthcare system.

Results A total of 130 patients were randomly allocated to OL-HDF $(n=67)$ and LF-HD $(n=63)$. The cost-utility ratio of OL-HDF versus LF-HD was Can $\$ 53,270$ per QALY gained over lifetime. This ratio was fairly robust in the sensitivity analysis. The cost-utility ratio was lower than that of LF-HD compared with no treatment (immediate death), which was Can\$93,008 per QALY gained. Conclusions High-efficiency OL-HDF can be considered a cost-effective treatment for ESRD in a Canadian setting. Further research is needed to assess cost effectiveness in other settings and healthcare systems.

\section{Key Points for Decision Makers}

The CONTRAST randomized controlled trial evaluated survival of dialysis patients on hemodiafiltration (HDF) compared with low-flux hemodialysis.

A subsequent simulation of costs and health outcomes concluded that HDF is not a cost-effective treatment. However, a major limitation of the CONTRAST study was a failure to deliver high-efficiency HDF.

St. Luc Hospital of the Centre Hospitalier de l'Université de Montréal (CHUM) was part of the CONTRAST study and consistently delivered highefficiency HDF. Analysis of this data shows that high-efficiency HDF can be considered a costeffective treatment in a Canadian setting.

This study shows that cost effectiveness of HDF is sensitive to the type of treatment delivered and the prevailing healthcare setting. 


\section{Introduction}

End-stage renal disease (ESRD) is the last stage of chronic kidney disease (CKD) and requires renal replacement therapy to keep patients alive, in the form of either dialysis treatment or a transplant. As of 2012, there were 41,252 people in Canada being treated for ESRD, with $58 \%$ $(n=23,814)$ on dialysis (of whom $82 \%$ were on hemodialysis and $18 \%$ on peritoneal dialysis) [1].

With an increasing number of patients in need of renal replacement therapy, many countries including Canada face the problem of managing ESRD in a sustainable way with limited resources. While, from a patient's perspective, the effectiveness of the therapy is of major importance, from a payer's and societal viewpoint, cost effectiveness also plays a critical role. Renal transplantation is clearly the more costeffective treatment for ESRD [2], but several limitations including the lack of an adequate number of available organs prevent a more widespread application. Since dialysis programs absorb a considerable amount of resources, several studies have evaluated alternative dialysis modalities within the scope of health technology assessment $[3,4]$.

Recently, an international parallel-group randomized clinical trial (RCT), the CONvective TRAnsport STudy (CONTRAST, NCT00205556) [5], was conducted with the aim to evaluate survival on an enhanced convective treatment, post-dilution on-line hemodiafiltration (OL-HDF), compared with low-flux hemodialysis (LF-HD) as standard of care. The trial did not show a significant survival advantage of OL-HDF [5] but suggested (and two other large trials suggested the same) that HDF had a beneficial effect on survival if a higher convection volume was provided [5-7].

From halfway through the CONTRAST trial, data were also collected on quality of life and resource consumption, the latter being multiplied with Dutch unit costs. Based on results of the trial, costs and health outcomes were simulated over a period of 5 years. The resulting cost-utility ratio based on year 2009 euros was $€ 287,679$ per qualityadjusted life-year (QALY) [8]. Given this finding, the authors concluded that, at present, OL-HDF cannot be considered a cost-effective treatment for patients with ESRD. However, a major limitation of the CONTRAST study was the failure to achieve the planned volume of post-dilution substitution fluid (19 L instead of the $24 \mathrm{~L}$ planned by protocol) in two-thirds of enrolled dialysis centers. As reported by Penne et al. [9], the practice patterns differed considerably between participating centers. According to a recent post hoc analysis [10], these differences result from center-specific practices regarding treatment time and blood flow rate, rather than characteristics of patients. Such differences can lead to varying levels of treatment effectiveness [5-7] and ultimately produce different cost-utility ratios. Moreover, cost differences of OLHDF depend also on the type of monitor used and the proprietary disposable material (tubing line and filter) for the OL-HDF modality [11].

St. Luc Hospital in Montréal, Canada, which belongs to the Centre hospitalier de l'Université de Montréal (CHUM) and has an annual caseload of approximately 140 hemodialysis patients as well as 20 peritoneal dialysis patients, was part of this international RCT. For the whole duration of the CONTRAST study, the CHUM conducted OL-HDF with the highest convection volume ( $>24 \mathrm{~L} / \mathrm{ses}$ sion) of all participating centers. Furthermore, since the introduction of hemodiafiltration in the CHUM in November 2007 (coinciding with the beginning of the CONTRAST study), proprietary cost data were collected in addition to CONTRAST data in the hospital for an independent cost analysis of the two treatments. At the end of the follow-up period of the CONTRAST study (March 2011), the CHUM decided to continue the study and enroll additional patients in the same manner until December 2013. With information on the additional patients, the CHUM aimed to decide whether to continue its OL-HDF program and whether to extend this treatment to all of their hemodialysis patients.

The purpose of this study was to assess the cost effectiveness of high-efficiency OL-HDF defined as OL-HDF performed with an optimal convection fluid volume (that is the sum of substitution fluid volume and net ultrafiltration) compared with LF-HD for patients with ESRD in this Canadian setting.

\section{Materials and Methods}

\subsection{Clinical Trial}

The present study was conducted within the scope of the CONTRAST study [5]. CONTRAST was a non-blinded parallel-group RCT (NCT00205556) that compared postdilution OL-HDF with LF-HD (both three times a week) on the basis of all-cause mortality. All CHUM CONTRAST study patients $(n=80)$ were randomized centrally by a computer-based randomization service (Julius Center University Medical Center, Utrecht, The Netherlands) into a 1:1 ratio for treatment with OL-HDF or continuation of LFHD, stratified per participating center (permuted blocks) [5]. Allocation concealment served to avoid selection bias by concealing what treatment the next patient would receive.

Starting in 2007, cost data were collected at the CHUM. The decision to continue the study after the end of the CONTRAST study was approved by the local medical ethics review board (http://www.clinicaltrials.gov; registered number CE10.253). Written informed consent to 
continue the study was requested from all trial participants who were still enrolled by December 31, 2010. Three patients, all on LF-HD, were unable to continue (censored) as their progressive cognitive impairment did not allow giving informed consent. For newly enrolled patients $(n=50)$, written informed consent was also obtained (prior to randomization). The study was conducted in accordance with the declaration of Helsinki and Good Clinical Practice Guidelines. Randomization procedure, monitoring, and data collection were kept the same as in the original trial period. Detailed information on study design and conduct as well as the inclusion and exclusion criteria can be found elsewhere [12].

\subsection{Dialysis Procedure}

Hemodiafiltration was performed in the online post-dilution mode. The following dialysis machines were used: 4008S with ONLINEplus ${ }^{\mathrm{TM}}$ (Fresenius Medical Care, Bad Homburg, Germany) for OL-HDF, and Integra (Gambro AB, Lund, Sweden) for LF-HD. The following synthetic dialyzers were used: Optiflux F200NR (Fresenius Medical Care, Bad Homburg, Germany) for all patients in the OLHDF arm, and Optiflux F18 (Fresenius Medical Care, Bad Homburg, Germany) for all patients in LF-HD arm. Ultrapure quality of water and dialysis fluids, defined as $<0.1 \mathrm{CFU} / \mathrm{mL}$ and $<0.03 \mathrm{IU} / \mathrm{mL}$, was used for all treatments. Microbiological tests of water and dialysis fluids were monitored each month in the CHUM, and microbiological assessments were compliant with reference quality levels in 2958 out of 2960 samples between November 2007 and December 2013.

Total duration of follow-up including the study period of CONTRAST and the period after CONTRAST was 74 months. Survival and economic data were assessed based on an 'intent to treat' analysis, thus analyzing patients according to the assigned treatment.

\subsection{Economic Evaluation}

The analysis was conducted from the perspective of the Quebec public healthcare system. Given this perspective, the study did not include productivity costs and copayments. On the other hand, the study included costs resulting from additional survival time as these are relevant from a healthcare system perspective.

We conducted a cost-effectiveness analysis using lifeyears gained as a measure of health benefits as well as a cost-utility analysis using QALYs. QALYs are the product of life-years and a representation of preference for different health states (preference weight or score). Preference weights are anchored on a scale from zero to one, where zero and one represent death and full health, respectively.
The incremental cost-effectiveness ratio (ICER) was determined by dividing the incremental costs (i.e., additional costs) of OL-HDF compared with LF-HD by the incremental (i.e., additional) life-years or QALYs gained. We conducted both an economic evaluation for the period of the RCT (74 months) based on data collected in the trial and an analysis over lifetime, thus following recommended practice [13]. For extrapolation beyond the trial period and in line with other recently published models $[8,14,15]$, we used a Markov (state transition) cohort model, thus assuming that transition probabilities do not depend on history. The Markov model used RCT data as well.

\subsection{Trial-Based Economic Evaluation}

\subsubsection{Cost Analysis}

The analysis considered costs both related and unrelated to renal disease including costs of dialysis side effects. Given the perspective of the evaluation, only direct costs were considered. Direct medical costs such as those related to dialysis staff and other medical staff, depreciation of dialysis equipment, water installation, disposables (dialyzers, blood lines, needles, etc.), routine diagnostics of patients, lab checks for water and dialysis fluid quality, and direct non-medical costs such as those related to transportation and laundry were obtained from the administration of the hospital. These costs are referred to as treatment costs. Treatment costs were assumed to be constant across time and patients. In reality, some variation may be possible (e.g., due to differences in the number of disposables), but these were considered negligible.

Pharmaceutical costs were calculated on the basis of a quarterly check of all prescriptions active during the month of the check. As a source of unit costs, we used the official price list issued by the healthcare authority in 2007 (list of medications covered by the basic prescription drug insurance plan, la Régie de l'Assurance-maladie du Québec, from June 2007) for all drugs available since the beginning of the study, and the official price at the time of introduction for all those subsequently authorized.

The cost of hospitalization was calculated using Quebec's diagnosis-related group (DRG) system, which assigns a relative measure of the volume of resources (Niveau d'Intensité Relative des Ressources Utilisées or NIRRU) to each patient at the time of discharge. Hospitalization costs were calculated by multiplying the NIRRU for each admission by the average hospitalization cost, which has a NIRRU of 1.0 (Can\$5319 in 2011/2012) [16-18].

We used a partitioned estimator proposed by Bang and Tsiatis [19] to calculate cumulative costs over the study period. This allows use of cost information from both censored and uncensored individuals. Accordingly, we 
partitioned the study period into 3-month intervals, estimated costs of all patients who were alive at the beginning of the interval, multiplied interval costs by the inverse probability of not being censored until the end of the interval, and calculated average cumulative costs by summing up these costs over the complete study period and dividing them by the cohort size in the first period. As recommended by Curtis et al. [20], we used a resampling method to estimate the standard error of average cumulative costs. Costs were adjusted to Canadian dollars in 2013 using the Canadian Consumer Price Index for Health and Personal Care [21].

\subsubsection{Survival Analysis}

Cumulative survival was analyzed using the Kaplan-Meier method, thus accounting for censored data. The Cox proportional-hazards model was used to estimate a hazard ratio of death with $95 \%$ confidence interval. The proportional hazards assumption was tested using the Schoenfeld residuals test.

\subsubsection{Quality-of-Life Analysis}

Preference weights were estimated using the 5-level version of the EuroQol-5D ${ }^{\mathrm{TM}}\left(\mathrm{EQ}-5 \mathrm{D}-5 \mathrm{~L}^{\mathrm{TM}}\right)$ questionnaire. The EQ-5D ${ }^{\mathrm{TM}}$ measures health-related quality of life in five dimensions: mobility, self care, usual activities, pain/ discomfort, and anxiety/depression [22]. Scores for the five dimensions are converted into preference weights by using country-specific value sets elicited from the general population. As the EQ-5D-5L $\mathrm{L}^{\mathrm{TM}}$ is relatively new, only a few country-specific value sets are available. A Canadian value set only exists for the three-level version of the EQ-5D ${ }^{\mathrm{TM}}$ [23]. Therefore, we used a UK value set for the base case and a US value set in a sensitivity analysis.

Quality-of-life data were obtained at the beginning of the trial as well as on an annual basis. Calculation of standard errors of mean OL-HDF and LF-HD preference weights accounted for between- and within-patient variation, thus considering correlation of individual quality-oflife data over time.

$p$ values $<0.05$ were considered statistically significant. We discounted both costs and effects at an annual rate of $3 \%$ [24].

\subsection{Model-Based Economic Evaluation}

\subsubsection{Model Description}

We constructed a Markov model to simulate the course of 1000 patients with ESRD (and treatment with OL-HDF or LF-HD). Patients were assumed to continue treatment with
OL-HDF or LF-HD over lifetime. A Markov model is an iterative process where patients are assumed to stay in one cycle (i.e., a defined health state) for a certain time and then make a transition to another cycle. Markov models are useful when a decision problem involves risk that is continuous over time, when the timing of events is important, and when important events may happen more than once. Our Markov model contains two health states, ESRD and death. Patients may transit to death at any time. Annual mortality was set to $15 \%$ (standard error $3 \%$ ) based on trial data (input data to the model are shown in Table 1). During each cycle, patients accumulate (quality-adjusted) life-years and costs. We chose a cycle length of 1 year for the two health states. The life-table method [25] was applied to both costs and life-years based on the assumption that transition events occur on average halfway through each 12-month cycle. The Markov model included 23 cycles to determine costs and effects over lifetime. After 23 cycles the proportion of patients still alive was less than $0.1 \%$ in both arms.

Annual healthcare costs for each of the two groups were calculated by dividing trial-based cumulative costs by the length of the trial period. For the base-case analysis, we discounted both costs and effects at an annual rate of $3 \%$ [24]. All calculations pertaining to the Markov model were done in Microsoft Excel (Microsoft Corporation, Redmond, WA, USA).

\subsubsection{Sensitivity Analysis}

To address uncertainty around the mean ICER, univariate sensitivity analyses were conducted where one variable was changed at a time while keeping all other variables constant at their mean or base-case value. We ran the analyses based on the upper and lower boundaries of the $95 \%$ confidence interval of the mean. To assess how a simultaneous change of several variables affects the costeffectiveness ratio, we performed a Monte-Carlo simulation, a type of multivariate sensitivity analysis. This technique runs a large number of simulations (here 1000) by repeatedly drawing samples from probability distributions of input variables. Thus, it provides a probability distribution for the output variables, that is, incremental costs, incremental effectiveness, and incremental cost-effectiveness ratios.

The annual probability of mortality and preference weights were assumed to follow a beta distribution because they are restricted to take on values between 0 and 1. The hazard ratio of death was assumed to follow normal distribution after logarithmic transformation. Cost data were assumed to follow a gamma distribution (reflecting the long right tail and restriction to positive values). 
Table 1 Data used for the model-based economic evaluation

\begin{tabular}{llll}
\hline Variable & Base case estimate & Range tested & References \\
\hline $\begin{array}{l}\text { Hazard ratio of death on OL-HDF } \\
\text { compared with LF-HD }\end{array}$ & 0.789 & $0.440-1.418$ & Trial data \\
Annual probability of death (LF-HD) & $15.2 \%$ & $9.5-21.0 \%$ & Trial data \\
Annual cost (LF-HD) & $€ 59,403$ & Obtained through trial-based Monte Carlo simulation & Trial data \\
Annual cost (OL-HDF) & $€ 59,308$ & Obtained through trial-based Monte Carlo simulation & Trial data \\
EQ-5D-5L & Trial data \\
EQ-5D-5L $\mathrm{L}^{\mathrm{TM}}$ index score for OL-HDF (UK value set) & 0.72 & $0.55-0.73$ & Trial data \\
Annual discount rate & $3 \%$ & $0.65-0.79$ & $0-7 \%$ \\
\hline
\end{tabular}

$E Q-5 D-5 L^{T M}$ 5-level version of the EuroQol-5D ${ }^{\mathrm{TM}}$ questionnaire, $L F-H D$ low-flux hemodialysis, $O L-H D F$ on-line hemodiafiltration

Given that the interpretation of negative ICERs is ambiguous, ICERs were transformed into net monetary benefits (NMB) [26] using the following equation:

$\mathrm{NMB}=\lambda \cdot \Delta E-\Delta C$

where $\lambda=$ maximum willingness-to-pay, $\Delta C=$ incremental costs, $\Delta E=$ incremental QALYs.

The decision rule used was to adopt the intervention in question if NMB was greater than zero. Given that the appropriate value of $\lambda$ is unknown, $\lambda$ was varied from Can $\$ 0$ to Can $\$ 200,000$. We generated a cost-effectiveness acceptability curve based on the distribution of NMB for each $\lambda$. A cost-effectiveness acceptability curve allows a decision maker to consider if OL-HDF is cost effective in relation to the maximum amount a decision maker is willing to pay for a QALY. At each ceiling value for the willingness to pay for a QALY, the cost-effectiveness acceptability curve shows the probability that OL-HDF is cost effective.

\section{Results}

\subsection{Study Population}

Eighty CHUM patients were originally enrolled in the CONTRAST study by using randomized 1:1 variable blocks. Patients were requested to remain in the study at the end of the follow-up. Only three patients, all on LF-HD, were no longer able to give an informed consent (censored) due to cognitive deterioration. An additional 50 patients were enrolled between 2011 and 2013 in the same manner (randomized 1:1, variable blocks). A total of 130 patients constituted the basis for this study and follow-up was maintained until December 31, 2013. Median follow-up was 2.53 years.

The baseline characteristics of the enrolled patients were similar in the two treatment arms and are reported in
Table 2. Treatment details during follow-up are provided in Table 3. Fourteen patients dropped out of the OL-HDF group because of transplantation $(n=6)$, transfer to another dialysis facility $(n=2)$, prolonged isolation $(n=4)$, or lack of compliance $(n=2)$. In the LF-HD group, 12 patients dropped out due to transplantation $(n=6)$, pre-dementia $(n=3)$, or transfer to another dialysis facility $(n=3)$. During follow-up, 23 patients in the OL-HDF group and 23 in the LF-HD group died.

\subsection{Clinical Trial}

OL-HDF led to non-significant improvements both in quality of life and survival. The hazard ratio of death of OL-HDF compared with LF-HD was 0.789 (95 \% confidence interval, 0.440-1.418). Schoenfeld residuals test did not suggest significant overall nonproportionality $(p=0.448)$. The non-significant improvement in quality of life (Table 4) can be partially attributed to differences which were present at baseline but based on data from $<50 \%$ of patients (i.e., 30 and 28 patients in the OL-HDF and LF-HD arm, respectively). Below, we also report the result of the extreme-case analysis assuming no improvement in quality of life.

\subsection{Trial-Based Economic Evaluation}

\subsubsection{Base Case}

Results of the trial-based analysis by arm are shown in Table 4. Treatment costs over 1 year for OL-HDF and LFHD were estimated to be Can $\$ 40,064$ and Can $\$ 42,017$, respectively (Table 4 reports costs over the complete trial period). Treatment costs per session for OL-HDF and LFHD were estimated to be Can $\$ 268.60$ and Can\$256.12, respectively, resulting in an additional treatment cost of OL-HDF compared with LF-HD of Can $\$ 12.48$ per session (Table 5). After treatment costs, hospitalization costs 
Table 2 Patient characteristics at baseline

\begin{tabular}{|c|c|c|}
\hline Characteristics & On-line hemodiafiltration & Low-flux hemodialysis \\
\hline Patients, $n$ & 67 & 63 \\
\hline Male gender, $n(\%)$ & $40(60)$ & $43(68)$ \\
\hline Age $(\text { years })^{a}$ & $64.2 \pm 14.1$ & $66.3 \pm 11.0$ \\
\hline Body mass index $\left(\mathrm{kg} / \mathrm{m}^{2}\right)^{\mathrm{a}}$ & $27.3 \pm 6.0$ & $27.6 \pm 6.6$ \\
\hline \multicolumn{3}{|l|}{ Primary renal disease, $n(\%)$} \\
\hline Glomerulonephritis & $17(25.3)$ & $9(14.3)$ \\
\hline Interstitial nephropathy & $6(9.0)$ & $2(3.2)$ \\
\hline Genetic disease & $5(7.5)$ & $2(3.2)$ \\
\hline Nephroangiosclerosis & $4(6.0)$ & $10(15.8)$ \\
\hline Diabetic nephropathy & $28(41.8)$ & $31(49.2)$ \\
\hline Unknown/others & $7(10.4)$ & $9(14.3)$ \\
\hline \multicolumn{3}{|l|}{ Comorbidities, $n(\%)$} \\
\hline Diabetes & $34(50.7)$ & $35(55.6)$ \\
\hline Coronary artery disease & $32(47.8)$ & $35(55.6)$ \\
\hline Peripheral vascular disease & $14(20.9)$ & $17(27.0)$ \\
\hline ESRD vintage (years) ${ }^{\mathrm{a}}$ & $3.20 \pm 4.04$ & $3.36 \pm 3.55$ \\
\hline \multicolumn{3}{|l|}{ Vascular access, $n(\%)$} \\
\hline AV-fistula & $42(63)$ & $34(54)$ \\
\hline Graft & $5(7)$ & $4(6)$ \\
\hline Catheter & $20(30)$ & $25(40)$ \\
\hline \multicolumn{3}{|l|}{ Biochemistry } \\
\hline B2-Microglobulin $(\mathrm{mg} / \mathrm{L})^{\mathrm{a}}$ & $34.0 \pm 16$ & $35.4 \pm 15.3$ \\
\hline Hemoglobin $(\mathrm{g} / \mathrm{L})^{\mathrm{a}}$ & $113.6 \pm 15.6$ & $112.0 \pm 11.2$ \\
\hline Phosphate $(\mathrm{mM} / \mathrm{L})^{\mathrm{a}}$ & $1.6 \pm 0.4$ & $1.7 \pm 0.5$ \\
\hline Calcium $(\mathrm{mM} / \mathrm{L})^{\mathrm{a}}$ & $2.2 \pm 0.2$ & $2.2 \pm 0.2$ \\
\hline Parathyroid hormone $(\mathrm{pg} / \mathrm{mL})^{\mathrm{a}}$ & $35.4 \pm 25.6$ & $42.2 \pm 35.0$ \\
\hline Albumin $(\mathrm{g} / \mathrm{L})^{\mathrm{a}}$ & $35.3 \pm 4.4$ & $35.6 \pm 3.4$ \\
\hline \multicolumn{3}{|l|}{ Treatment parameters } \\
\hline Session length $(\mathrm{h})^{\mathrm{a}}$ & $3.76 \pm 0.37$ & $3.80 \pm 0.31$ \\
\hline Dialysis frequency $2 /$ week, $n(\%)$ & $2(3)$ & None \\
\hline Dialysis frequency $3 /$ week, $n(\%)$ & $65(97)$ & $63(100)$ \\
\hline Blood flow $(\mathrm{mL} / \mathrm{min})^{\mathrm{a}}$ & $368.83 \pm 46.23$ & $350.7 \pm 28.7$ \\
\hline 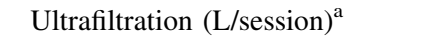 & $2.67 \pm 0.49$ & $2.99 \pm 1.18$ \\
\hline Single-pool Kt/V urea ${ }^{a}$ & $1.64 \pm 0.37$ & $1.5 \pm 0.25$ \\
\hline
\end{tabular}

$E S R D$ end-stage renal disease

${ }^{\text {a }}$ Mean \pm standard deviation

represent the second highest cost category (Table 4). Figure 1 depicts the hospitalization length and the number of hospitalizations by cause. Infectious complications were the main reason both for the number of hospitalizations and the number of days spent in the hospital.

Disregarding costs of the additional survival time on OL-HDF, discounted total per-patient costs over the trial period amount to Can $\$ 203,082$ and Can $\$ 203,629$ for OLHDF and LF-HD, respectively. That is, assuming no survival benefit, OL-HDF leads to savings of Can\$547. ICERs are shown in Table 6. Based on gains in quality of life, the cost-per-QALY-gained ratio is lower than the cost-per-lifeyear-gained ratio.

\subsubsection{Sensitivity Analysis}

Using a US value set for EQ-5D ${ }^{\mathrm{TM}}$ health states results in a cost-utility ratio of Can\$35,250 per QALY gained.

\subsection{Model-Based Economic Evaluation}

\subsubsection{Base Case}

As shown in Table 6, the model-based ICERs are of comparable relative magnitude to those of the trial-based evaluation. That is, based on gains in quality of life, the cost-per-QALY-gained ratio is lower than the cost-per-life- 
Table 3 Patient treatment details during follow-up (mean \pm standard deviation)

\begin{tabular}{|c|c|c|c|c|}
\hline & $\begin{array}{l}\text { Follow-up } \\
\text { (months) }\end{array}$ & $\begin{array}{l}\text { On-line } \\
\text { hemodiafiltration }\end{array}$ & $\begin{array}{l}\text { Low-flux } \\
\text { hemodialysis }\end{array}$ & $\begin{array}{l}p \text { value for difference } \\
\text { of means }\end{array}$ \\
\hline \multirow[t]{4}{*}{ Treatment time $(\min )$} & $1-3$ & $225.7 \pm 21.9$ & $228.1 \pm 18.5$ & 0.498 \\
\hline & $10-12$ & $226.2 \pm 19.2$ & $227.9 \pm 17.5$ & 0.702 \\
\hline & $22-24$ & $229.4 \pm 19.8$ & $234.2 \pm 12.1$ & 0.278 \\
\hline & $34-36$ & $232.5 \pm 13.9$ & $225.0 \pm 16.4$ & 0.373 \\
\hline \multirow[t]{4}{*}{ Proportion of patients on 3 sessions/week (\%) } & $1-3$ & 97.0 & 100 & 0.264 \\
\hline & $10-12$ & 97.4 & 100 & 0.551 \\
\hline & $22-24$ & 96.8 & 100 & 0.544 \\
\hline & $34-36$ & 87.5 & 100 & 0.571 \\
\hline \multirow[t]{4}{*}{$\mathrm{Kt} / \mathrm{V}$} & $1-3$ & $1.76 \pm 0.34$ & $1.54 \pm 0.44$ & 0.02 \\
\hline & $10-12$ & $1.89 \pm 0.32$ & $1.54 \pm 0.23$ & $<0.001$ \\
\hline & $22-24$ & $1.96 \pm 0.31$ & $1.57 \pm 0.23$ & $<0.001$ \\
\hline & $34-36$ & $1.94 \pm 0.31$ & $1.59 \pm 0.22$ & $<0.001$ \\
\hline \multirow[t]{4}{*}{$\beta 2$-Microglobulin clearance (mL/min) } & $1-3$ & $67.5 \pm 13.2$ & & \\
\hline & $10-12$ & $69.1 \pm 15.4$ & & \\
\hline & $22-24$ & $65.2 \pm 13.2$ & & \\
\hline & $34-36$ & $76.5 \pm 22.9$ & & \\
\hline \multirow[t]{4}{*}{ Blood flow (mL/min) } & $1-3$ & $385.8 \pm 46.5$ & $358.0 \pm 33.7$ & $<0.001$ \\
\hline & $10-12$ & $410.5 \pm 52.4$ & $367.3 \pm 26.7$ & $<0.001$ \\
\hline & $22-24$ & $428.5 \pm 37.7$ & $383.1 \pm 32.3$ & $<0.001$ \\
\hline & $34-36$ & $415.2 \pm 40.9$ & $378.9 \pm 27.0$ & $<0.001$ \\
\hline \multirow[t]{4}{*}{ Convective volume (L/sessions) } & $1-3$ & $26.6 \pm 7.1$ & & \\
\hline & $10-12$ & $27.9 \pm 7.7$ & & \\
\hline & $22-24$ & $27.3 \pm 8.6$ & & \\
\hline & $34-36$ & $22.7 \pm 11.9$ & & \\
\hline \multirow[t]{4}{*}{ Darbepoietin alfa dose ( $\mu \mathrm{g} /$ week) } & $1-3$ & $47.6 \pm 36.8$ & $50.3 \pm 48.6$ & 0.714 \\
\hline & $10-12$ & $51.0 \pm 44.9$ & $60.3 \pm 49.4$ & 0.297 \\
\hline & $22-24$ & $49.3 \pm 43.4$ & $67.1 \pm 60.9$ & 0.148 \\
\hline & $34-36$ & $59.2 \pm 58.1$ & $64.9 \pm 61.8$ & 0.759 \\
\hline \multirow[t]{4}{*}{ Sevelamer dose (g/day) } & $1-3$ & $2.34 \pm 2.93$ & $3.42 \pm 3.37$ & 0.055 \\
\hline & $10-12$ & $2.14 \pm 2.88$ & $4.05 \pm 3.71$ & 0.003 \\
\hline & $22-24$ & $1.99 \pm 2.72$ & $4.62 \pm 3.69$ & 0.001 \\
\hline & $34-36$ & $2.49 \pm 2.98$ & $4.71 \pm 4.70$ & 0.062 \\
\hline Hospitalization rate (days/patient-year) & All & $16.8 \pm 28.7$ & $22.2 \pm 46.1$ & 0.416 \\
\hline Hospitalization rate (n/patient-year) & All & $1.06 \pm 1.36$ & $1.36 \pm 1.59$ & 0.248 \\
\hline
\end{tabular}

year-gained ratio. Furthermore, the cost-utility ratio of LFHD compared with immediate death (assuming that ESRD patients without LF-HD would die immediately) is Can\$93,008 per QALY gained (306,826/3.30).

\subsubsection{Sensitivity Analysis}

Using a US value set results in a cost-utility ratio of Can\$54,978 per QALY gained. Using a UK value set for EQ-5D ${ }^{\mathrm{TM}}$ health states and assuming the same level of quality of life in both arms increases the cost-utility ratio to Can\$82,072 per QALY gained.
In the univariate sensitivity analysis, the variable with largest impact on the cost-per-QALY-gained ratio of OLHDF versus LF-HD was the hazard ratio of death on OLHDF compared with LF-HD. Note that the upper limit leads to a positive ratio which is based on a reduction both in costs and QALYs (Table 7).

Figure 2 shows a scatter plot of incremental costs and QALYs of OL-HDF compared with LF-HD. As displayed, the ratio of incremental QALYs to incremental costs is fairly robust as pairs of incremental costs and QALYs fall on a straight constant. In $28 \%$ of replications, OL-HDF was less effective (led to fewer QALYs) than LF-HD. The 
Table 4 Results of the trialbased analysis by arm. Costs are undiscounted, in 2013 Canadian dollars, and refer to one patient over the trial period of $\sim 6.2$ years

\begin{tabular}{|c|c|c|c|}
\hline Variable & On-line hemodiafiltration & Low-flux hemodialysis & $p$ value \\
\hline Treatment costs, Can\$ & $259,453^{\mathrm{a}}$ & $247,398^{\mathrm{a}}$ & $<0.001$ \\
\hline Hospitalization costs, Can\$ & $70,717(940)$ & $70,219(1013)$ & NS \\
\hline Drug costs, Can\$ & $36,059(112)$ & $49,196(190)$ & $<0.001$ \\
\hline Total costs, Can\$ & $366,229(957)$ & $366,813(1057)$ & NS \\
\hline EQ-5D-5L $\mathrm{L}^{\mathrm{TM}}$ index score (UK value set) & $0.72(0.03)$ & $0.64(0.05)$ & NS \\
\hline EQ-5D-5L ${ }^{\mathrm{TM}}$ index score (US value set) & $0.79(0.02)$ & $0.73(0.03)$ & NS \\
\hline
\end{tabular}

Standard errors of the mean are given in parentheses

$E Q-5 D-5 L^{T M}$ 5-level version of the EuroQol-5D ${ }^{\mathrm{TM}}$ questionnaire, $N S$ not significant

${ }^{a}$ No uncertainty in estimates was assumed

\begin{tabular}{lcr}
\hline Item & $\begin{array}{c}\text { Cost/session OL } \\
\text { HDF, Can\$ (\%) }\end{array}$ & $\begin{array}{l}\text { Cost/session LF-HD, } \\
\text { Can\$ (\%) }\end{array}$ \\
\hline Labour & $155.54(58.0)$ & $155.54(60.7)$ \\
Disposables & $49.85(18.6)$ & $42.60(16.6)$ \\
Intravenous drugs & $19.03(7.1)$ & $19.03(7.4)$ \\
Laboratory checks & $5.34(2.0)$ & $5.34(2.1)$ \\
Vascular access & $3.46(1.3)$ & $3.46(1.4)$ \\
Dialysis machines: supplies, monitoring, depreciation, etc. & $19.39(7.2)$ & $15.86(6.2)$ \\
Water treatment: supplies, monitoring, depreciation, etc. & $4.03(1.5)$ & $2.52(1.0)$ \\
Laundry, hygiene, and safety & $5.14(1.9)$ & $5.14(2.0)$ \\
Transportation & $3.45(1.3)$ & $3.45(1.3)$ \\
Other & $3.17(1.1)$ & $3.17(1.2)$ \\
Total & $268.40(100)$ & $256.12(100)$ \\
\hline
\end{tabular}

$L F-H D$ low-flux hemodialysis, $O L-H D F$ on-line hemodiafiltration
Table 5 Breakdown of the additional treatment cost of OLHDF compared with LF-HD

\section{Discussion}

interval of the hazard ratio of death on OL-HDF compared with LF-HD is larger than one. Figure 3 shows the costeffectiveness acceptability curve, which considers uncertainty in cost effectiveness. The probability of cost effectiveness is largest at a willingness to pay of approximately Can\$90,000 per QALY gained. Increasing willingness to pay further leads to a decrease in the probability of cost effectiveness because the joint density of incremental costs and effects in the southwest quadrant of the cost-effectiveness plane (Fig. 2) is excluded for no longer being cost effective.

\subsubsection{Internal Validation}

Internal model validation was checked as follows. According to Gandjour and Gafni [27], the ratio of downstream costs to effects of both interventions is the same at a single time point regardless of how effective the intervention is. When also assuming the same annual costs and quality of life, both interventions should yield the same ICER. Following this procedure we indeed obtained this result, thus confirming the internal validity of our model.
Based on the results of the Canadian arm of the CONTRAST study, this study simulated costs and health benefits of high-efficiency OL-HDF versus LF-HD over lifetime. It shows a cost-utility ratio of Can $\$ 53,270$ per QALY gained of OL-HDF versus LF-HD. As shown by the sensitivity analysis, the ratio of incremental QALYs to incremental costs is fairly robust. The cost-utility ratio is lower than that of LF-HD compared with no treatment (immediate death), which is Can\$93,008 per QALY gained (Canada does not have an explicit cost-effectiveness threshold [28]). Hence, based on the assumption that ESRD patients without LF-HD would die immediately, OL-HDF weakly dominates LF-HD in the base case. That is, OLHDF has an ICER that is lower than that of LF-HD and at the same time it is more effective than LF-HD. Based on the principle of weak dominance, OL-HDF can be considered a cost-effective treatment for ESRD in this Canadian setting. This result is also obtained based on the costeffectiveness acceptability curve presented in Fig. 3. Using Can\$93,008 per QALY gained (the ICER of LF-HD) as the willingness to pay for a QALY, the probability of cost 
Fig. 1 Length and number of hospitalizations by cause for all patients

\section{Length of stay (\% of days)}

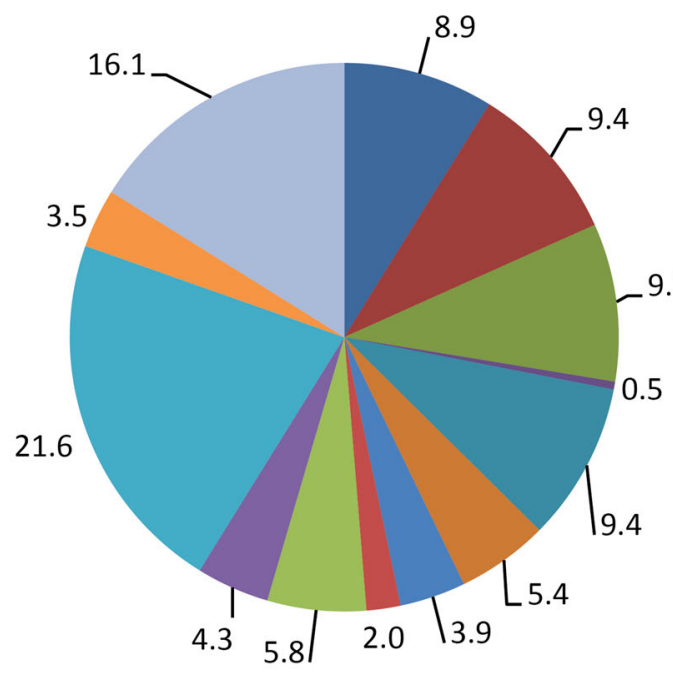

- vascular access infection

- respiratory infection

घother infection

non-heart-related edema

neripheral vascular disease

neart ischemic disease, myocardial infarction

E heart failure

cerebral vascular disease

Eastro-intestinal bleeding

cancer

loss of autonomy

voluntary stop of treatment

wher causes

No. of Hospitalizations (\%)

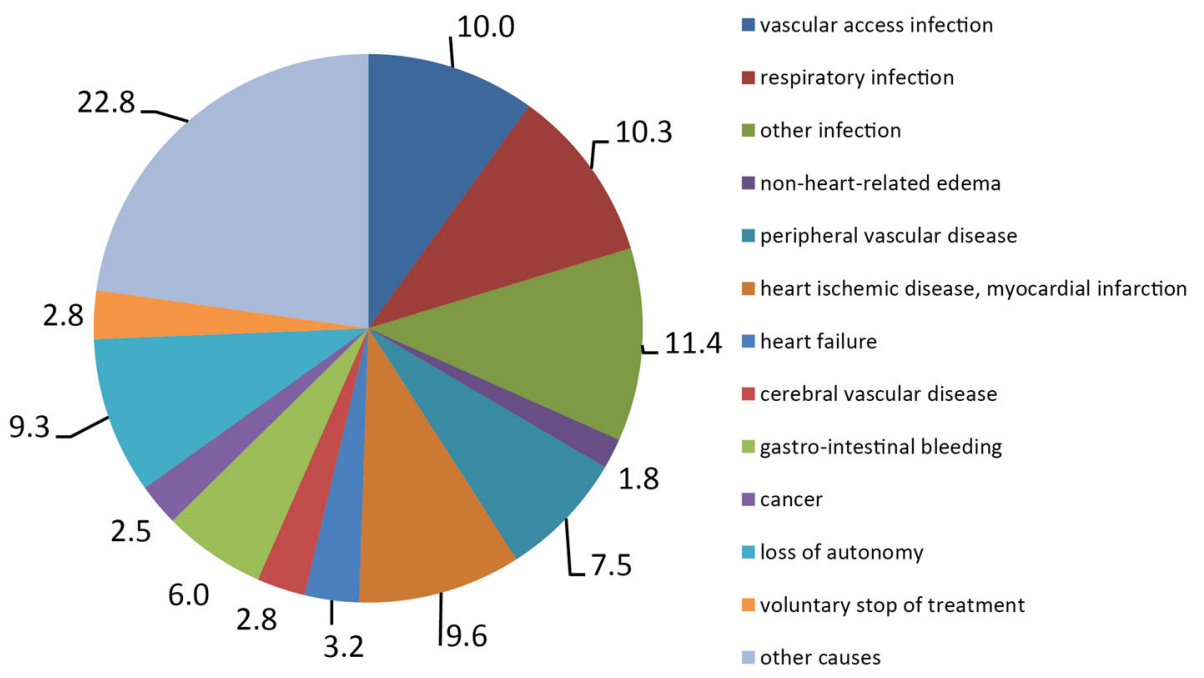

effectiveness is above $90 \%$. A word of caution: it cannot be excluded that OL-HDF is less effective than LF-HD as the upper bound of the $95 \%$ confidence interval of the hazard ratio of death on OL-HDF compared with LF-HD is larger than one. Furthermore, our result, strictly speaking, only applies to in-center patients for whom home dialysis is not an option. Otherwise, the analysis would need to include home dialysis as a comparator.

One may consider no treatment as an unrealistic alternative for patients who could be treated either with hemodialysis or extended conservative or supportive therapy. Yet, considering conservative management as a comparator of hemodialysis would likely increase the cost- effectiveness ratio of hemodialysis even further. Also, using no treatment as a comparator of hemodialysis is quite common in the health economics literature. In fact, it is the cost-effectiveness ratio of hemodialysis compared with no treatment which is often used as a benchmark of societal willingness to pay in the academic literature [29]. We followed this practice and thus also used it as a benchmark for the cost-effectiveness ratio of OL-HDF.

Our model has the same structure as the cost-utility analysis based on the original CONTRAST study [8], i.e., a Markov model containing two health states, dialysis and death. Two other recently published models $[14,15]$ consider in addition transitions between different dialysis 
Table 6 Discounted incremental costs, effects, and cost effectiveness of on-line hemodiafiltration (OL-HDF) versus low-flux hemodialysis (LF-HD)

\begin{tabular}{llllll}
\hline & Costs & $\begin{array}{l}\text { Life- } \\
\text { years }\end{array}$ & QALYs & $\begin{array}{l}\text { Incremental costs } \\
\text { per life-year gained } \\
\text { (OL-HDF vs LF-HD) }\end{array}$ & $\begin{array}{l}\text { Incremental costs per } \\
\text { QALY gained (OL-HDF } \\
\text { vs LF-HD) }\end{array}$ \\
\hline \multicolumn{2}{l}{$\begin{array}{l}\text { Trial-based analysis } \\
\text { OL-HDF } \quad 220,018\end{array}$} & 4.01 & 2.87 & 53,153 & 32,112 \\
LF-HD & 203,629 & 3.70 & 2.36 & & 53,270 \\
Model-based analysis & & & & \\
OL-HDF & 368,177 & 6.21 & 4.45 & 58,840 & \\
LF-HD & 306,826 & 5.17 & 3.30 & & \\
\hline
\end{tabular}

Costs are in 2013 Canadian dollars and are rounded

$Q A L Y$ quality-adjusted life-year
Table 7 Univariate sensitivity analysis

\begin{tabular}{lc}
\hline Variable & $\begin{array}{l}\text { Incremental costs per QALY } \\
\text { gained over lifetime }\end{array}$ \\
\hline Annual discount rate & \\
Lower limit (0 \%) & 56,034 \\
Upper limit (7 \%) & 49,832 \\
Annual probability of death on LF-HD \\
Lower limit (10 \%) \\
Upper limit (21 \%) \\
Hazard ratio of death for OL-HDF compared with LF-HD \\
Lower limit (0.440) & 72,129 \\
Upper limit (1.418) & 145,696 \\
\hline
\end{tabular}

Costs are in 2013 Canadian dollars

$L F-H D$ low-flux dialysis, $O L-H D F$ on-line hemodiafiltration, $Q A L Y$ quality-adjusted life-year

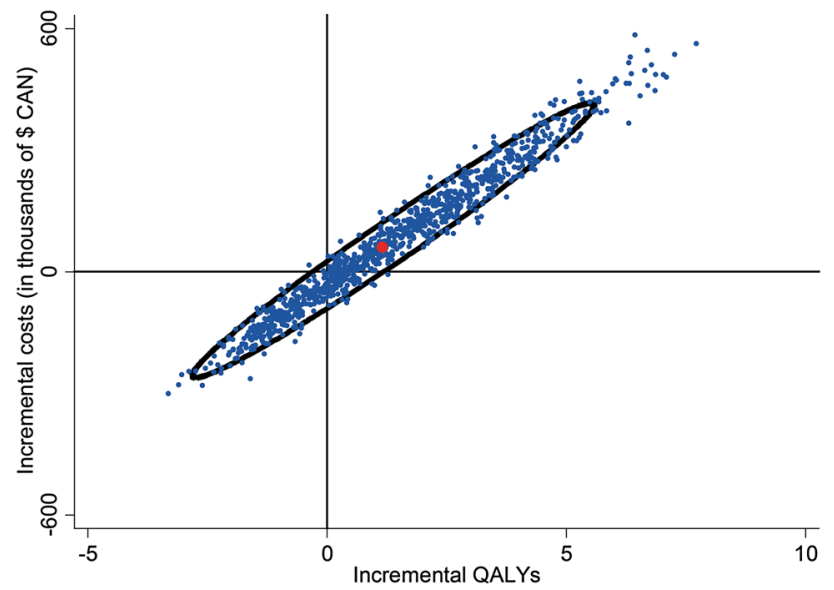

Fig. 2 Scatter plot of incremental costs and quality-adjusted lifeyears (QALYs) of on-line hemodiafiltration (OL-HDF) compared with low-flux hemodialysis (LF-HD). Plotted are a $95 \%$ confidence ellipse (black line) and the mean cost-effect pair (red dot) modes, between dialysis and transplantation, as well as between dialysis with and without complications. Yet, in our trial, patients did not switch between OL-HDF and LFHD. Furthermore, the number of dropouts due to transplantation was the same in both groups and thus cancels out from the calculation of the ICER. Finally, while we included the cost of dialysis side effects, we did not model it through the probability of side effects due to availability of patient-level data.

The trial population was representative for the Canadian ESRD population for the variables age, sex, and primary kidney disease [30] and the two arms were well balanced with respect to baseline characteristics. In this study, nonsignificant improvements in quality of life and survival may be the result of a lack of power due to small sample size. However, considering even non-significant changes in health for the calculation of ICERs is consistent with the concept of 'irrelevance of inference' [31]. Accordingly, rules of inference are arbitrary and entirely irrelevant for decisions based on economic evaluations.

The result of this study differs from that for the Dutch evaluation of the CONTRAST study, which, based on year 2009 euros, reported a cost-utility ratio of $€ 287,679$ per QALY gained over a period of 5 years [8] (corresponding to Can $\$ 420,357$ as of August 7, 2014). A major reason is the larger QALY gain by OL-HDF in our study, resulting from larger gains both in quality of life and survival compared with the original CONTRAST study. Indeed, one can hypothesize that larger improvement of patient outcomes is likely attributable to clinical practices of this center, i.e., achievement of a higher convective volume was able to optimize overall treatment effectiveness.

The estimate for the hazard ratio in this sample $(0.79)$ falls in the range of a recently published estimate $(0.84$; $95 \%$ confidence interval $0.73-0.96$ ) from a meta-analysis [32] of six studies comparing OL-HDF with low- or high- 
Fig. 3 Cost-effectiveness acceptability curve. $Q A L Y$ quality-adjusted life-year

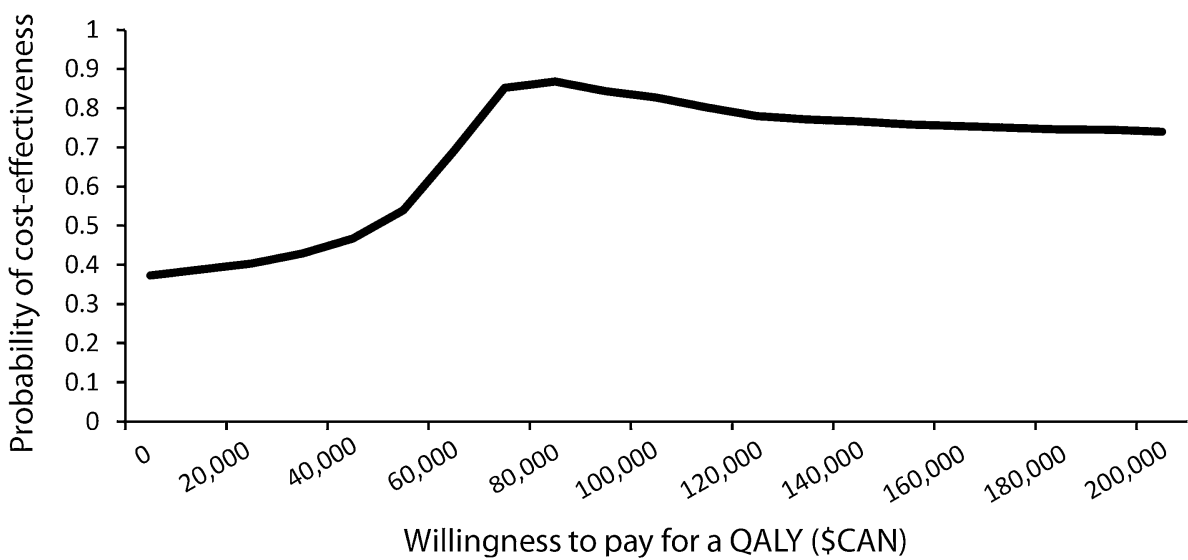

flux HD (four studies were on post-dilution [5-7, 33], one on pre-dilution [34], and one on mixed [35] OL-HDF). Furthermore, the hazard ratio in this study is lower than for the original CONTRAST study (0.95) [5], indicating improved survival. The latter result can potentially be explained by the higher mean convective volume of $27.4 \pm 7.3 \mathrm{~L} /$ session as compared with a mean of $19.7 \mathrm{~L} /$ session in the original CONTRAST study [10]. According to Sehgal et al. [36], dialysis dose for low molecular weight substances is inversely correlated to morbidity. Thus, patients with lower dialysis dose have higher hospitalization rates. The same relationship holds for the convective dose [7, 37]. For example, in the study of Maduell et al. [7], a convective dose in the range of $23-24 \mathrm{~L} / \mathrm{session}$ in patients assigned to on-line OL-HDF was associated with a lower all-cause hospitalization rate.

A recent systematic review [38] including 226 utility estimates from dialysis patients (the majority of whom were receiving hemodialysis) reported a preference weight of 0.70 , very close to the estimates in the current study of 0.72 and 0.64 for OL-HDF and LF-HD, respectively (when applying the UK value set).

At CHUM, the additional treatment cost of OL-HDF compared with LF-HD was estimated to be Can $\$ 12.48$ per session, ensuring 'ultrapure dialysate' to patients both on OL-HDF and LF-HD. In 2002, Beerenhout et al. [39] estimated this extra cost to be in the range of $€ 6$ to $€ 21$ when comparing OL-HDF with high-flux 'ultrapure' HD and in the range of $€ 17$ to $€ 32$ when comparing it with LFHD. In the meantime, the difference in costs between lowand high-flux membranes has decreased and microbiological testing requirements have been reduced.

Our study shows that spending on pharmaceuticals was $14 \%$ lower for patients on OL-HDF. Lower costs of pharmaceuticals mainly resulted from significantly lower doses of phosphate binders. Doses of rHu-erythropoietin and erythropoiesis-stimulating agents were also lower, in line with the result of prospective observational studies [40,
41] and RCTs [42], but the difference did not reach the level of statistical difference. It should be stressed that the level of hemoglobin correction was comparable for patients on OL-HDF and LF-HD (e.g., 11.4 vs $11.0 \mathrm{~g} / \mathrm{dL}$ in the third month of follow-up and $11.5 \mathrm{vs} 11.0 \mathrm{~g} / \mathrm{dL}$ in the twelfth month of follow-up for OL-HDF and LF-HD, respectively). In the CONTRAST study [5], costs for $\mathrm{rHu}-$ erythropoietin were very similar in both treatment groups.

The following limitations of the study have to be taken into consideration. First, the reference treatment of the CONTRAST study was LF-HD. Actually, according to market survey data, only $5 \%$ of the dialyzers sold in Canada are in the low-flux range. However, since the cost difference between low-flux and high-flux dialyzers is very small today and the hazard ratio estimated by our study is in the range of the estimations of Maduell et al. [7] for high-flux HD as a comparator, this limitation should not have significantly biased the results. Also, less costly dialysis modalities exist in Canada for more autonomous and healthier (or less comorbid) patients for whom home dialysis is an option [43]. As a second limitation, while we included costs of hospitalizations for diseases related and unrelated to kidney disease, we did not consider costs of unplanned outpatient visits. However, these costs may be the same in both arms and thus cancel out. Third, due to the nature of the treatments, both patients enrolled and medical personnel were not blinded to treatment. While quality-oflife improvements by OL-HDF could therefore be attributed to a placebo effect, this effect would also occur outside the trial setting and thus should be accounted for. Fourth, it cannot be excluded that an imbalance in baseline characteristics had an impact on results. Yet, even if the real survival benefit were smaller than shown by the study, results would also be favorable for OL-HDF. That is, when assuming no survival benefit, our discounted cost analysis suggests savings through OL-HDF. Finally, renal units considering the introduction of OL-HDF would face implementation costs in terms of modifying dialysis 
delivery to a new system with potential capital, operational, and training costs. As is typical for cost-effectiveness analyses, these implementation costs were not included. Also, renal units may be hesitant to introduce OL-HDF for the reason that the survival benefit of OL-HDF carries some uncertainty or is not strongly recommended by guidelines yet. Therefore, our results primarily support those renal units which have already switched or have made the decision to switch to OL-HDF.

In summary, in the Canadian arm of the CONTRAST study, high efficiency OL-HDF was found to be a costeffective treatment for ESRD compared with LF-HD. Studies in different healthcare systems and regulatory environments are required to confirm the result.

Compliance with Ethical Standards R. Lévesque received support from Amgen Canada with no conflict of interest for this study. D. Marcelli, A. Grassmann and B. Canaud are employees of Fresenius Medical Care Deutschland, producer of dialysis equipment for both dialysis therapies under comparison. M. Grooteman received an unrestricted research grant from Fresenius Medical Care Netherlands and received symposia sponsorship from Sanofi-Aventis and Fresenius with no conflict of interest for this study. H. Cardinal, M.-L. Caron, M. Bots, P. Blankestijn, and M. Nubé have no conflict of interest with the subject matter or materials discussed in the manuscript. A. Gandjour received a grant from Fresenius Medical Care Deutschland for this work. No writing assistance was utilized in the production of this manuscript.

The study was approved by the local medical ethics review board (CE10.253). It was conducted in accordance with the declaration of Helsinki and Good Clinical Practice Guidelines. Informed consent was obtained from all individual participants included in the study.

Author Contributions CONTRAST study design and reporting: R. Lévesque, H. Cardinal, M. Grooteman, M. Bots, P. Blankenstijn, M. Nubé. Research idea and study design: R. Lévesque, D. Marcelli, B. Canaud, A. Gandjour. Data management: R. Lévesque, M-L. Caron, D. Marcelli. Statistical analysis: D. Marcelli, A. Gandjour. Data interpretation and writing of the manuscript: R. Lévesque, D. Marcelli, H. Cardinal, M.-L. Caron, M. Grooteman, M. Bots, P. Blankstijn, M. Nubé, A. Grassmann, B. Canaud, A. Gandjour. Guarantor for the overall content: D. Marcelli.

Open Access This article is distributed under the terms of the Creative Commons Attribution-NonCommercial 4.0 International License (http://creativecommons.org/licenses/by-nc/4.0/), which permits any noncommercial use, distribution, and reproduction in any medium, provided you give appropriate credit to the original author(s) and the source, provide a link to the Creative Commons license, and indicate if changes were made.

\section{References}

1. Webster G, Wu J, Williams B, Ivis F, de Sa E, Hall N. Canadian organ replacement register annual report: treatment of end-stage organ failure in Canada 2003-2012. Ottawa: Canadian Institute for Health Information; 2014.

2. Howard K, Salkeld G, White S, McDonald S, Chadban S, Craig $\mathrm{JC}$, et al. The cost-effectiveness of increasing kidney transplantation and home-based dialysis. Nephrology (Carlton). 2009;14(1):123-32.

3. Mowatt G, Vale L, Perez J, Wyness L, Fraser C, MacLeod A, et al. Systematic review of the effectiveness and cost-effectiveness, and economic evaluation, of home versus hospital or satellite unit haemodialysis for people with end-stage renal failure. Health Technol Assess. 2003;7(2):1-174.

4. Sesso R, da Silva CB, Kowalski SC, Manfredi SR, Canziani ME, Draibe SA, et al. Dialysis care, cardiovascular disease, and costs in end-stage renal disease in Brazil. Int $\mathrm{J}$ Technol Assess Health Care. 2007;23(1):126-30.

5. Grooteman MP, van den Dorpel MA, Bots ML, Penne EL, van der Weerd NC, Mazairac AH, et al. Effect of online hemodiafiltration on all-cause mortality and cardiovascular outcomes. J Am Soc Nephrol. 2012;23(6):1087-96.

6. Ok E, Asci G, Toz H, Ok ES, Kircelli F, Yilmaz M, et al. Mortality and cardiovascular events in online haemodiafiltration (OL-HDF) compared with high-flux dialysis: results from the Turkish OL-HDF Study. Nephrol Dial Transplant. 2013;28(1):192-202.

7. Maduell F, Moreso F, Pons M, Ramos R, Mora-Macia J, Carreras $\mathrm{J}$, et al. High-efficiency postdilution online hemodiafiltration reduces all-cause mortality in hemodialysis patients. J Am Soc Nephrol. 2013;24(3):487-97.

8. Mazairac AH, Blankestijn PJ, Grooteman MP, Penne EL, van der Weerd NC, den Hoedt $\mathrm{CH}$, et al. The cost-utility of haemodiafiltration versus haemodialysis in the Convective Transport Study. Nephrol Dial Transplant. 2013;28(7):1865-73.

9. Penne EL, van der Weerd NC, Bots ML, van den Dorpel MA, Grooteman MP, Levesque R, et al. Patient- and treatment-related determinants of convective volume in post-dilution haemodiafiltration in clinical practice. Nephrol Dial Transplant. 2009;24(11):3493-9.

10. Chapdelaine I, Mostovaya IM, Blankestijn PJ, Bots ML, van den Dorpel MA, Levesque R, et al. Treatment policy rather than patient characteristics determines convection volume in online post-dilution hemodiafiltration. Blood Purif. 2014;37(3):229-37.

11. Lebourg L, Amato S, Toledano D, Petitclerc T, Creput C. Online hemodiafiltration: is it really more expensive? Nephrol Ther. 2013;9(4):209-14.

12. Penne EL, Blankestijn PJ, Bots ML, van den Dorpel MA, Grooteman MP, Nube MJ, et al. Effect of increased convective clearance by on-line hemodiafiltration on all cause and cardiovascular mortality in chronic hemodialysis patients-the Dutch CONvective TRAnsport STudy (CONTRAST): rationale and design of a randomised controlled trial [ISRCTN38365125]. Curr Control Trials Cardiovasc Med. 2005;6(1):8.

13. Sculpher MJ, Claxton K, Drummond M, McCabe C. Whither trial-based economic evaluation for health care decision making? Health Econ. 2006;15(7):677-87.

14. Liu FX, Treharne C, Arici M, Crowe L, Culleton B. High-dose hemodialysis versus conventional in-center hemodialysis: a costutility analysis from a UK payer perspective. Value Health. 2015;18(1):17-24.

15. Treharne C, Liu FX, Arici M, Crowe L, Farooqui U. Peritoneal dialysis and in-centre haemodialysis: a cost-utility analysis from a UK payer perspective. Appl Health Econ Health Policy. 2014;12(4):409-20.

16. Lemay A, Handfield D, Mireault J. Évaluation de la valeur prédictive de l'indice d'intensité des ressources par épisode de soins construit à partir de la structure de facturation de l'état du Maryland. Montrèal: AHQ; 1999.

17. Lemay A, Aumont I, Hel C. Le réseau en 4 questions, un regard ciblé sur la performance. Association québecoise des éstablissements de santé et des services sociaux (AQESS). 2013. http:// www.aqess.qc.ca. Accessed 5 Dec 2014. 
18. Mode d'allocation des ressources financières 2006-2007 aux hopitaux deu Québec pour les patients hospitalisés et en chirurgie d'un jour. Ministère de la Santè et des Services Sociaux. 2007. http://www.oiiaq.org/documents/file/mode=allocationressources-hopitauy.pdf. Accessed 5 Dec 2014.

19. Bang H, Tsiatis A. Estimating medical costs with with censored data. Biometrika. 2000;87:329-43.

20. Curtis LH, Hammill BG, Eisenstein EL, Kramer JM, Anstrom KJ. Using inverse probability-weighted estimators in comparative effectiveness analyses with observational databases. Med Care 2007;45(10 Supl 2):S103-7.

21. Statistics Canada. 2014. http://www.statcan.gc.ca. Accessed 31 July 2014.

22. The EuroQol Group. EuroQol-a new facility for the measurement of health-related quality of life. Health Policy 1990;16(3):199-208.

23. Bansback N, Tsuchiya A, Brazier J, Anis A. Canadian valuation of EQ-5D health states: preliminary value set and considerations for future valuation studies. PLoS One. 2012;7(2):e31115.

24. Gold M. Panel on cost-effectiveness in health and medicine. Med Care. 1996;34(12 Suppl):DS197-9.

25. Barendregt JJ. The half-cycle correction: banish rather than explain it. Med Decis Mak. 2009;29(4):500-2.

26. Stinnett AA, Mullahy J. Net health benefits: a new framework for the analysis of uncertainty in cost-effectiveness analysis. Med Decis Mak. 1998;18(2 Suppl):S68-80.

27. Gandjour A, Gafni A. Internal validation of models with several interventions. Eur J Health Econ. 2013;14(6):901-9.

28. Paris V, Belloni A. Value in pharmaceutical pricing-country profile: Canada. Paris: OECD; 2014. http://www.oecd.org/ canada/Value-in-Pharmaceutical-Pricing-Canada.pdf. Accessed 22 April 2014.

29. Winkelmayer WC, Weinstein MC, Mittleman MA, Glynn RJ, Pliskin JS. Health economic evaluations: the special case of endstage renal disease treatment. Med Decis Mak. 2002;22(5):417-30.

30. Traitement du stade terminal de l'insuffisance organique au Canada, de 2002-2011 - rapport annuel du registre canadien des insuffisances et des transplantations d'organes (2012). Institut Canadien d'information sur la santé. 2014.

31. Claxton K. The irrelevance of inference: a decision-making approach to the stochastic evaluation of health care technologies. J Health Econ. 1999;18(3):341-64.
32. Mostovaya IM, Blankestijn PJ, Bots ML, Covic A, Davenport A, Grooteman MP, et al. Clinical evidence on hemodiafiltration: a systematic review and a meta-analysis. Semin Dial. 2014;27(2):119-27.

33. Locatelli F, Mastrangelo F, Redaelli B, Ronco C, Marcelli D, La $\mathrm{GG}$, et al. Effects of different membranes and dialysis technologies on patient treatment tolerance and nutritional parameters. The Italian Cooperative Dialysis Study Group. Kidney Int. 1996;50(4):1293-302.

34. Locatelli F, Altieri P, Andrulli S, Bolasco P, Sau G, Pedrini LA, et al. Hemofiltration and hemodiafiltration reduce intradialytic hypotension in ESRD. J Am Soc Nephrol. 2010;21(10):1798-807.

35. Wizemann V, Kulz M, Techert F, Nederlof B. Efficacy of haemodiafiltration. Nephrol Dial Transplant. 2001;16(Suppl 4):27-30.

36. Sehgal AR, Dor A, Tsai AC. Morbidity and cost implications of inadequate hemodialysis. Am J Kidney Dis. 2001;37(6):1223-31.

37. Canaud B, Bowry SK. Emerging clinical evidence on online hemodiafiltration: does volume of ultrafiltration matter? Blood Purif. 2013;35(1-3):55-62.

38. Wyld M, Morton RL, Hayen A, Howard K, Webster AC. A systematic review and meta-analysis of utility-based quality of life in chronic kidney disease treatments. PLoS Med. 2012;9(9):e1001307.

39. Beerenhout CH, Kooman JP, Luik AJ, Jeuken-Mertens SG, van der Sande FM, Leunissen KM. Optimizing renal replacement therapy - a case for online filtration therapies? Nephrol Dial Transplant. 2002;17(12):2065-70.

40. Bonforte G, Grillo P, Zerbi S, Surian M. Improvement of anemia in hemodialysis patients treated by hemodiafiltration with highvolume on-line-prepared substitution fluid. Blood Purif. 2002;20(4):357-63.

41. Lin CL, Huang CC, Yu CC, Wu CH, Chang CT, Hsu HH, et al. Improved iron utilization and reduced erythropoietin resistance by on-line hemodiafiltration. Blood Purif. 2002;20(4):349-56.

42. Vaslaki L, Major L, Berta K, Karatson A, Misz M, Pethoe F, et al. On-line haemodiafiltration versus haemodialysis: stable haematocrit with less erythropoietin and improvement of other relevant blood parameters. Blood Purif. 2006;24(2):163-73.

43. Klarenbach SW, Tonelli M, Chui B, Manns BJ. Economic evaluation of dialysis therapies. Nat Rev Nephrol. 2014;10(11):644-52. 